\title{
FIXED POINT AND EXTENDED COUPLED FIXED POINT THEOREMS FOR MULTI-VALUED CONTRACTIONS WITH RESPECT TO THE POMPEIU FUNCTIONAL
}

\author{
IULIA COROIAN
}

Received 07 November, 2019

\begin{abstract}
In this paper, we will prove some fixed point results using a Pompeiu type metric on $P_{c l}(X)$, for multi-valued operators satisfying two conditions: contractivity and monotonicity. The approach is based on a fixed point theorem for a multi-valued operator in the setting of a b-metric space. Several qualitative properties(well-posedness, Ulam-Hyers stability) are also obtained. In the second part of this paper, we will consider the extended coupled fixed point problem for a multi-valued operator. We will consider the problem of the existence of the solutions. Data dependence and well-posedness of the extended coupled fixed point problem are also discussed.
\end{abstract}

2010 Mathematics Subject Classification: 47H10; 54H25

Keywords: multi-valued operator, fixed point, ordered metric space, extended coupled fixed point, data dependence, well-posedness, Ulam-Hyers stability, limit shadowing

\section{INTRODUCTION}

Nadler's contraction principle [14] is an extension to the multi-valued case of the classical Banach's contraction principle. There are many applications of these results, mainly in the theory of operator equations and inclusions, see [2], [4], [6], [9] and [12]. On the other hand, several theorems were given for the so-called extended coupled fixed point problem([3], [17],[18]).

We will define first the notion of b-metric.

Definition 1 (Bakhtin [1], Czerwik [7]). Let $X$ be a nonempty set and let $s \geq 1$ be a given real number. A functional $d: X \times X \rightarrow \mathbb{R}_{+}$is said to be a $b$-metric with constant $s$ if all the axioms of the metric take place with the following modification of the triangle inequality property:

$$
d(x, z) \leq s[d(x, y)+d(y, z)], \text { for all } x, y, z \in X .
$$

Under the above hypotheses, the pair $(X, d)$ is called a $b$-metric space with constant s. 
In the framework of a $b$-metric space, a set $Y \subset X$ is said to be closed if for any sequence $\left(x_{n}\right)_{n \in \mathbb{N}}$ in $Y$ which converges (with respect to $d$ ) to an element $x$, we have $x \in Y$. The notions of bounded or compact sets are defined in a similar way to the case of usual metric spaces. For example, the set $Y \subset X$ is said to be bounded if its diameter $\delta(Y):=\sup _{x, y \in Y} d(x, y)$ is finite, while $Y$ is called compact if every sequence sequence $\left(x_{n}\right)_{n \in \mathbb{N}}$ in $Y$ has a convergent subsequence in $Y$.

Let $(X, d)$ be a $b$-metric space and $\mathcal{P}(\mathrm{X})$ be the set of all subsets of $\mathrm{X}$. We denote

$$
\begin{aligned}
P(X) & :=\{Y \in \mathcal{P}(X) \mid Y \neq \varnothing\}, \\
P_{c l}(X) & :=\{Y \in \mathcal{P}(X) \mid Y \text { is closed }\}, \\
P_{c p}(X) & :=\{Y \in \mathcal{P}(X) \mid Y \text { is compact }\} .
\end{aligned}
$$

Let $(X, d)$ be a $b$-metric space. If $T: X \rightarrow P(X)$ is a multi-valued operator, then $x \in X$ is called fixed point for $\mathrm{T}$ if and only if

$$
x \in T(x) .
$$

We denote by $\operatorname{Fix}(T)$ the fixed point set of $T$ and by $\operatorname{SFix}(T)$ the set of all strict fixed points of $T$, i.e., the elements $x \in X$ such that $T(x)=\{x\}$.

If $X, Y$ are two nonempty sets and $T: X \rightarrow P(Y)$, then we will denote by

$$
\operatorname{Graph}(T):=\{(x, y) \in X \times Y: y \in T(x)\}
$$

the graph of $T$.

In the context of a $b$-metric space $(X, d)$ the following generalized functionals are used in the main sections of the paper.

(1) The gap functional generated by $d$ :

$$
D_{d}: P(X) \times P(X) \rightarrow \mathbb{R}_{+}, D_{d}(A, B)=\inf \{d(a, b) \mid a \in A, b \in B\} .
$$

(2) The excess generalized functional:

$$
e_{d}: P(X) \times P(X) \rightarrow \mathbb{R}_{+} \cup\{\infty\}, e_{d}(A, B)=\sup \left\{D_{d}(a, B) \mid a \in A\right\} .
$$

(3) The Hausdorff-Pompeiu generalized functional :

$$
H_{d}: P(X) \times P(X) \rightarrow \mathbb{R}_{+} \cup\{\infty\}, H_{d}(A, B)=\max \left\{e_{d}(A, B), e_{d}(B, A)\right\} .
$$

(4) The Pompeiu generalized functional:

$$
H_{d}^{+}: P(X) \times P(X) \rightarrow \mathbb{R}_{+} \cup\{\infty\}, H_{d}^{+}(A, B):=\frac{1}{2}\left\{e_{d}(A, B)+e_{d}(B, A)\right\} .
$$

Definition 2 ([17]). Let $X$ be a nonempty set, let " $\leq$ " be a partial order on $X$ and $d$ be a $b$-metric on $X$ with constant $s \geq 1$. Then the triple $(X, \leq, d)$ is called an ordered $b$-metric space if:

i. " $\leq$ " is a partial order on $X$;

ii. $d$ is a $b$-metric on $X$ with constant $s \geq 1$; 
iii. if $\left(x_{n}\right)_{n \in \mathbb{N}}$ is a monotone increasing sequence in $X$ and $x_{n} \rightarrow x^{*} \in X$, then $x_{n} \leq x^{*}$, for all $n \in \mathbb{N}$;

iv. if $\left(y_{n}\right)_{n \in \mathbb{N}}$ is a monotone decreasing sequence in $X$, and $y_{n} \rightarrow y^{*} \in X$, then $y_{n} \geq y^{*}$, for all $n \in \mathbb{N}$.

Definition 3 ([17]). Let $(X, \leq)$ be a partially ordered set. Then, the partial order " $\leq$ " induces on the product space $X \times X$ the following partial order:

$$
\text { for }(x, y),(u, v) \in X \times X,(x, y) \leq_{p}(u, v) \Leftrightarrow x \leq u, y \geq v .
$$

Definition 4 ([18]). Let $(X, d),(Y, d)$ be two complete $b$-metric spaces, $T_{1}: X \times$ $Y \rightarrow P(X)$ and $T_{2}: X \times Y \rightarrow P(Y)$ be two multi-valued operators. Then, by definition, a extended coupled fixed point for $T_{1}$ and $T_{2}$ is a pair $\left(x^{*}, y^{*}\right) \in X \times Y$ satisfying

$$
\left\{\begin{array}{l}
x^{*} \in T_{1}\left(x^{*}, y^{*}\right) \\
y^{*} \in T_{2}\left(x^{*}, y^{*}\right) .
\end{array}\right.
$$

We will denote by $\operatorname{CFix}\left(T_{1}, T_{2}\right)=\left\{(x, y) \in X \times Y \mid x \in T_{1}(x, y), y \in T_{2}(x, y)\right\}$ the solution set for problem (P1).

In this paper, using a Pompeiu type metric on $P_{c l}(X)$, we will prove some fixed point results for multi-valued operators satisfying a combined condition (contractivity and monotonicity). Then, as applications, we will obtain some existence results for a general system of operator inclusions. Several qualitative properties(data dependence, well-posedness) are also obtained.

\section{Preliminaries}

We will start this section by recalling some useful notion and results.

Lemma 1 ([18]). Let $(X, d)$ be a b-metric space with constant $s \geq 1, A, B \in P(X)$ and $q>1$. Then, for every $a \in A$ there exists $b \in B$ such that $e_{d}(A, B) \leq q d(a, b)$.

Definition 5. Let $(X, \preceq)$ be a partially ordered set and $A, B \in P(X)$. We will denote:

a) $A \leq_{s t} B \Leftrightarrow \forall a \in A, \forall b \in B$ we have $a \preceq b$;

b) $A \leq_{w k} B \Leftrightarrow \forall a \in A, \exists b \in B$ such that $a \preceq b$.

Remark 1. Notice that if $A, B, C \in P(X)$, then $A \leq_{s t} B$ and $B \leq_{s t} C$ implies $A \leq_{s t} C$. The same property also holds for $\leq_{w k}$.

Definition 6. Let $(X, \preceq)$ a partially ordered set and $G: X \times X \rightarrow P(X)$. We say that $G$ has the strict mixed monotone property with respect to the partial order " $\preceq$, if the following conditions hold:

(1) $x_{0} \preceq x_{1} \Rightarrow G\left(x_{0}, y\right) \leq_{s t} G\left(x_{1}, y\right), \forall y \in X$;

(2) $y_{0} \succeq y_{1} \Rightarrow G\left(x, y_{0}\right) \leq_{s t} G\left(x, y_{1}\right), \forall x \in X$. 
Remark 2. Let $(X, d)$ be a $b$-metric space with constant $s \geq 1$ and $Z:=X \times X$. Then the functional $\tilde{d}: Z \times Z \rightarrow \mathbb{R}_{+}$defined by

$$
\tilde{d}((x, y),(u, v))=d(x, u)+d(y, v), \text { for all }(x, y),(u, v) \in Z
$$

is a $b$-metric on $Z$ with the same constant $s \geq 1$ and if $(X, d)$ is a complete $b$-metric space, then $(Z, \tilde{d})$ is a complete $b$-metric space, too.

Moreover, for $x, y \in X, A, B, U, V \in P(X)$ we have:

$$
\begin{gathered}
D_{\tilde{d}}((x, y), A \times B)=D_{d}(x, A)+D_{d}(y, B), \\
e_{\tilde{d}}(U \times V, A \times B)=e_{d}(U, A)+e_{d}(V, B), \\
H_{\tilde{d}}(U \times V, A \times B) \leq H_{d}(U, A)+H_{d}(V, B), \\
H_{\tilde{d}}^{+}(U \times V, A \times B)=H_{d}^{+}(U, A)+H_{d}^{+}(V, B) .
\end{gathered}
$$

Additionally, by the properties of the gap functional $D_{d}$, if $(x, y) \in X \times X$ and $A, B \in$ $P_{c l}(X)$, then

$$
D_{\tilde{d}}((x, y), A \times B)=0 \text { if and only if }(x, y) \in A \times B .
$$

Definition 7. Let (X,d) be a $b$-metric space with constant $s \geq 1$. A multi-valued operator $T: X \rightarrow P_{b, c l}(X)$ is called $H^{+}$-contraction with constant $k$ if:

(1) there exists a fixed real number $0<k<1$ such that, for every $x, y \in X$, we have

$$
H_{d}^{+}(T(x), T(y)) \leq k d(x, y),
$$

(2) for every $x \in X$ and $y \in T(x)$ we have

$$
D_{d}(y, T(y)) \leq H_{d}^{+}(T(x), T(y)) .
$$

In particular, a multivalued mapping $T: X \rightarrow P_{c l}(X)$ is called $\left(H^{+}, \alpha\right)$-Lipschitz if $\alpha>0$ and

$$
H_{d}^{+}(T(x), T(y)) \leq \alpha d(x, y), \quad \forall x, y \in X .
$$

Definition 8. Let $(X, \preceq)$ be a partially ordered set and $T: X \rightarrow P(X)$ be a multivalued operator. We say that $T$ is strong increasing (respectively strong decreasing) on $X$ if, for every $x, y \in X$ with $x \preceq y$ we have that $T(x) \leq_{s t} T(y)$ (respectively $T(x) \geq_{s t}$ $T(y))$.

Lemma 2 (R. Miculescu, A. Mihail [13]). Every sequence $\left(x_{n}\right)_{n \in \mathbb{N}}$ of elements from b-metric space $(X, d)$ of constant $s$, having the property that there exists $\gamma \in$ $[0,1)$ such that

$$
d\left(x_{n+1}, x_{n}\right) \leq \gamma d\left(x_{n}, x_{n-1}\right), \forall n \in \mathbb{N},
$$


is Cauchy. Moreover

$$
d\left(x_{n+1}, x_{n+k}\right) \leq \gamma^{n} \frac{S}{1-\gamma} d\left(x_{0}, x_{1}\right), \forall n, k \in \mathbb{N}^{*}, \text { where } S=\sum_{n \geq 1} \gamma^{2 n \log _{\gamma} s+2^{n-1}} .
$$

Lemma 3. Let $(X, d)$ be a b-metric space with constant $s \geq 1$ and $T: X \rightarrow P_{b, c l}(X)$ be a $\left(H_{d}^{+}, k\right)$ - Lipschitz. Then:

(1) Thas closed graph in $X \times X$;

(2) $T$ is $H_{d}^{+}$-l.s.c on $X$;

(3) $T$ is $H_{d}^{+}$-u.s.c on $X$;

(4) If, additionally $T$ has compact values, then $T$ is l.s.c..

Proof. (1) Let $\left(x_{n}, y_{n}\right) \subset X \times X$ such that $\left(x_{n}, y_{n}\right) \underline{d}(x, y)$, when $n \rightarrow \infty$ and $y_{n} \in T\left(x_{n}\right)$, for all $n \in \mathbb{N}$.

It follows that:

$$
\begin{aligned}
D(y, T(x)) & \leq s\left[d\left(y, y_{n}\right)+D\left(y_{n}, T(x)\right)\right] \leq \\
& \leq s\left[d\left(y, y_{n}\right)+H_{d}\left(T\left(x_{n}\right), T(x)\right)\right] \leq \\
& \leq s\left[d\left(y, y_{n}\right)+2 H_{d}^{+}\left(T\left(x_{n}\right), T(x)\right)\right] \leq \\
& \leq s\left[d\left(y, y_{n}\right)+2 \alpha d\left(x_{n}, x\right)\right], \text { for all } n \in \mathbb{N} .
\end{aligned}
$$

Let us consider $n \rightarrow \infty$ and we obtain $D(y, T(x)) \leq 0$. Thus $y \in T(x)$ which means that $\mathrm{T}$ has a closed graph.

(2) Let $x \in X$ such that $x_{n} \rightarrow x$. We have:

$$
\begin{aligned}
e_{d}\left(T(x), T\left(x_{n}\right)\right) & \leq H_{d}\left(T(x), T\left(x_{n}\right)\right) \leq 2 H_{d}^{+}\left(T(x), T\left(x_{n}\right)\right) \leq \\
& \leq 2 \alpha d\left(x, x_{n}\right) \rightarrow 0 .
\end{aligned}
$$

Then, $\mathrm{T}$ is $H_{d}^{+}$-1.s.c. on X.

(3) Using the relation:

$$
\begin{aligned}
e_{d}\left(T\left(x_{n}\right), T(x)\right) & \leq H\left(T\left(x_{n}\right), T(x)\right) \leq 2 H_{d}^{+}\left(T\left(x_{n}\right), T(x)\right) \leq \\
& \leq 2 \alpha d\left(x, x_{n}\right) \rightarrow 0,
\end{aligned}
$$

we obtain that $\mathrm{T}$ is $H_{d}^{+}$-u.s.c. on $\mathrm{X}$.

(4) The conclusion follows by the fact that any $H_{d}^{+}$-1.s.c multivalued operator with compact values is l.s.c..

Lemma 4 (Cauchy's Lemma). Let $\left(a_{n}\right)_{n \in \mathbb{N}}$ and $\left(b_{n}\right)_{n \in \mathbb{N}}$ be two sequences of nonnegative real numbers, such that $\sum_{p=0}^{\infty} a_{p}<+\infty$ and $\lim _{n \rightarrow \infty} b_{n}=0 . \quad$ Then, $\lim _{n \rightarrow \infty}\left(\sum_{p=0}^{n} a_{n-p} b_{p}\right)=0$. 


\section{FIXED POINT THEOREMS FOR MULTI-VALUED CONTRACTIONS WITH RESPECT TO A POMPEIU TYPE METRIC}

In this paper, we will present some fixed point theorems for a multi-valued operators in b-metric spaces. See also [5], [11], [10] and [15] for related results.

Theorem 1. Let $(X, \preceq, d)$ be an ordered b-metric space with constant $s \geq 1$ such that, the b-metric d is complete. Let $T: X \rightarrow P_{c l}(X)$ be a multi-valued strong increasing operator with closed graph. Assume that:

(1) there exists $k<1$ such that

$$
H_{d}^{+}(T(x), T(y)) \leq k d(x, y), \forall x, y \in X, x \preceq y,
$$

(2) for every $x \in X$ and $y \in T(x)$ we have,

$$
D_{d}(y, T(y)) \leq H_{d}^{+}(T(x), T(y)),
$$

(3) there exists an element $x_{0} \in X$ such that

$$
x_{0} \leq_{w k} T\left(x_{0}\right) .
$$

Then,

a) $\operatorname{Fix}(T) \neq \varnothing$.

b) there exists a sequence of successive approximation for $T$ starting from $x_{0}$ such that

$$
d\left(x_{n+1}, x^{*}\right) \leq \frac{\beta^{n} S s}{1-\beta} d\left(x_{0}, x_{1}\right), \forall n \in \mathbb{N}, \text { where } \beta>0, \text { with } k<\beta<1 .
$$

Proof. a) Let $x_{0} \in X$ such that $x_{0} \leq_{w k} T\left(x_{0}\right)$. By (3.3) we get that there exists $x_{1} \in T\left(x_{0}\right)$ such that $x_{0} \preceq x_{1}$.

Using (3.2) we obtain that,

$$
D_{d}\left(x_{1}, T\left(x_{1}\right)\right) \leq H_{d}^{+}\left(T\left(x_{0}\right), T\left(x_{1}\right)\right) \leq k d\left(x_{0}, x_{1}\right) .
$$

For $k<\beta<1$ the relation (3.4) becames

$$
D_{d}\left(x_{1}, T\left(x_{1}\right)\right)<\beta d\left(x_{0}, x_{1}\right) .
$$

Thus, there exists $x_{2} \in T\left(x_{1}\right)$ such that

$$
d\left(x_{1}, x_{2}\right)<\beta d\left(x_{0}, x_{1}\right) .
$$

Since $x_{0} \preceq x_{1}$ and $\mathrm{T}$ is a multi-valued strong increasing operator, we get that

$$
T\left(x_{0}\right) \leq_{s t} T\left(x_{1}\right) \text {. Thus } x_{1} \preceq x_{2} \text {. }
$$

By (3.2) we get that,

$D_{d}\left(x_{2}, T\left(x_{2}\right)\right) \leq H_{d}^{+}\left(T\left(x_{1}\right), T\left(x_{2}\right)\right) \leq k d\left(x_{1}, x_{2}\right)<\beta d\left(x_{1}, x_{2}\right)<\beta^{2} d\left(x_{0}, x_{1}\right)$.

By induction, we obtain a sequence $\left(x_{n}\right)_{n \in \mathbb{N}} \in X$ with the following properties:

(1) $x_{n+1} \in T\left(x_{n}\right), \forall n \in \mathbb{N}$,

(2) $d\left(x_{n}, x_{n+1}\right)<\beta d\left(x_{n-1}, x_{n}\right)$, 
(3) $d\left(x_{n}, x_{n+1}\right)<\beta^{n} d\left(x_{0}, x_{1}\right), \forall n \in \mathbb{N}$.

By Lemma 2 we get that $\left(x_{n}\right)_{n \in \mathbb{N}}$ is Cauchy in $(X, d)$. Then, there exists $x^{*} \in X$ such that $\lim _{n \rightarrow \infty} x_{n}=x^{*}$. Since T has closed graph in $(X, d)$ we obtain that $x^{*} \in$ Fix $(\mathrm{T})$.

b) By Lemma 2 we have

$$
d\left(x_{n+1}, x_{n+k}\right) \leq \beta^{n} \frac{S}{1-\beta} d\left(x_{0}, x_{1}\right), \forall n, k \in \mathbb{N}^{*}, \text { where } S=\sum_{n \geq 1} \gamma^{2 n \log _{\gamma} s+2^{n-1}} .
$$

By (1) we have that $x_{n} \rightarrow x^{*}, n \rightarrow \infty$. Then,

$$
\frac{1}{s} d\left(x_{n+1}, x^{*}\right) \leq d\left(x_{n+1}, x_{n+k}\right)+d\left(x_{n+k}, x^{*}\right) \leq \frac{\beta^{n} S}{1-\beta} d\left(x_{0}, x_{1}\right)+d\left(x_{n+k}, x^{*}\right) .
$$

Letting $k \rightarrow \infty$, we get $\frac{1}{s} d\left(x_{n+1}, x^{*}\right) \leq \beta^{n} \frac{S}{1-\beta} d\left(x_{0}, x_{1}\right)$. Hence $d\left(x_{n+1}, x^{*}\right) \leq$ $\beta^{n} \frac{S s}{1-\beta} d\left(x_{0}, x_{1}\right)$.

The next result is a corollary of the previous theorem for the global case, where it isn't necessary to suppose that the operator $T$ has to be with closed graph.

Corollary 1. Let $(X, d)$ be a complete b-metric space with constant $s \geq 1$. Let $T: X \rightarrow P_{c l}(X)$ be a multi-valued operator. Assume that:

(1) there exists $k<1$ such that,

$$
H_{d}^{+}(T(x), T(y)) \leq k d(x, y), \forall x, y \in X,
$$

(2) for every $x \in X$ and $y \in T(x)$ we have,

$$
D_{d}(y, T(y)) \leq H_{d}^{+}(T(x), T(y))
$$

Then $\operatorname{Fix}(T) \neq \varnothing$.

The following result is about the uniqueness of the solution for the fixed point problem (P).

Theorem 2. Suppose that all the hypotheses of Theorem 1 are satisfied. Additionally, we suppose there exists $x^{*} \in X$ such that $T\left(x^{*}\right)=\left\{x^{*}\right\}$ and for every $\bar{x} \in$ Fix $(T)$ we have $\bar{x} \preceq x^{*}$ or $x^{*} \preceq \bar{x}$. Then Fix $(T)=\operatorname{SFix}(T)=\left\{x^{*}\right\}$.

Proof. Let $x^{*} \in X$ such that $T\left(x^{*}\right)=\left\{x^{*}\right\}$. Then, for every $\bar{x} \in$ Fix $(T)$ we have $\bar{x} \preceq x^{*}$ or $x^{*} \preceq \bar{x}$. Then,

$$
d\left(\bar{x}, x^{*}\right)=D_{d}\left(\bar{x}, T\left(x^{*}\right)\right) \leq H_{d}^{+}\left(T(\bar{x}), T\left(x^{*}\right)\right) \leq k d\left(\bar{x}, x^{*}\right) .
$$

Thus, $(1-k) d\left(\bar{x}, x^{*}\right) \leq 0$ which means that $d\left(\bar{x}, x^{*}\right)=0$ and $\bar{x}=x^{*}$. Thus, $\operatorname{Fix}(T)=$ $\operatorname{SFix}(T)=\left\{x^{*}\right\}$. 
We have the following global version of the Theorem 2.

Corollary 2. Let $(X, \preceq, d)$ be a complete $b$-metric space with constant $s \geq 1$. Let $T: X \rightarrow P_{c l}(X)$ be a multi-valued operator. Assume that:

(1) there exists $k<1$ such that,

$$
H_{d}^{+}(T(x), T(y)) \leq k d(x, y), \forall x, y \in X,
$$

(2) for every $x \in X$ and $y \in T(x)$ we have,

$$
D_{d}(y, T(y)) \leq H_{d}^{+}(T(x), T(y)),
$$

(3) there exists $x^{*} \in X$ such that $T\left(x^{*}\right)=\left\{x^{*}\right\}$.

Then Fix $(T)=\operatorname{SFix}(T)=\left\{x^{*}\right\}$.

In the next part of this section, we will present some properties of that fixed point problem $(\mathrm{P})$. The first one is the data dependence problem for the fixed point problem (P).

Theorem 3. Let $(X, d)$ be a complete b-metric space with constant $s \geq 1$ and let $T: X \rightarrow P_{c l}(X), S: X \rightarrow P(X)$ be two multi-valued operators. We suppose that:

(1) there exists $k<\frac{1}{2 s}$ such that

$$
H_{d}^{+}(T(x), T(y)) \leq k d(x, y), \forall x, y \in X,
$$

(2) there exists $x^{*} \in X$ such that $T\left(x^{*}\right)=\left\{x^{*}\right\}$,

(3) there exists $\eta>0$ such that

$$
e_{d}(T(x), S(x)) \leq \eta, \forall x \in X .
$$

Then, $e_{d}(\operatorname{Fix}(T), \operatorname{Fix}(S)) \leq \frac{s \eta}{1-2 s k}$.

Proof. By (1) and (2) we get that Fix $(T)=\operatorname{SFix}(T)=\left\{x^{*}\right\}$. Let $u^{*} \in X$ such that $u^{*} \in S\left(u^{*}\right)$ and $x^{*} \in X$ such that $T\left(x^{*}\right)=\left\{x^{*}\right\}$. We have,

$$
\begin{aligned}
d\left(u^{*}, x^{*}\right) & =D_{d}\left(u^{*}, T\left(x^{*}\right)\right) \leq \\
& \leq s\left[D_{d}\left(u^{*}, T\left(u^{*}\right)\right)+e_{d}\left(T\left(u^{*}\right), T\left(x^{*}\right)\right)\right] \\
& \leq s\left[e_{d}\left(S\left(u^{*}\right), T\left(u^{*}\right)\right)+H_{d}\left(T\left(u^{*}\right), T\left(x^{*}\right)\right)\right] \leq \\
& \leq s\left[\eta+2 H_{d}^{+}\left(T\left(u^{*}\right), T\left(x^{*}\right)\right)\right] \leq \\
& \leq s\left[\eta+2 k d\left(u^{*}, x^{*}\right)\right] .
\end{aligned}
$$

Then, $d\left(u^{*}, x^{*}\right) \leq \frac{s \eta}{1-2 s k}$.

Since $u^{*} \in$ Fix $(S)$ are arbitrary chosen, we obtain that, $e_{d}(\operatorname{Fix}(T), F i x(S)) \leq \frac{s \eta}{1-2 s k}$. 
The next problem we intend to study is the well-posedness of the fixed point problem $(\mathrm{P})$.

Definition 9. We consider the fixed point problem (P). By definition, $(\mathrm{P})$ is wellposed for $T$ with respect to $D_{d}$ if:

(1) $\operatorname{Fix}(T)=\left\{x^{*}\right\}, x^{*} \in X$.

(2) if there exists a sequence $u_{n} \in X$ with $D_{d}\left(u_{n}, T\left(u_{n}\right)\right) \rightarrow 0$, then $d\left(u_{n}, x^{*}\right) \rightarrow 0$ as $n \rightarrow \infty$

Theorem 4. We suppose that all the hypotheses of Corollary 2 take place. Then the fixed point problem $(\mathrm{P})$ is well-posed for $T$ with respect to $D_{d}$.

Proof. Since the multi-valued operator T satisfies the hypotheses of Corollary 1, we get that $\mathrm{T}$ has a unique fixed point, i.e., $\operatorname{Fix}(T)=\left\{x^{*}\right\}$ where $x^{*} \in X$. Then we have, $T\left(x^{*}\right)=\left\{x^{*}\right\}$.

Let $u_{n} \subset X$, with $D_{d}\left(u_{n}, T\left(u_{n}\right)\right) \rightarrow 0$ as $n \rightarrow \infty$.

Then we have,

$$
\begin{aligned}
d\left(u_{n}, x^{*}\right) & =D_{d}\left(u_{n}, T\left(x^{*}\right)\right) \leq \\
& \leq s\left[D_{d}\left(u_{n}, T\left(u_{n}\right)\right)+e_{d}\left(T\left(u_{n}\right), T\left(x^{*}\right)\right)\right] \leq \\
& \leq s\left[D_{d}\left(u_{n}, T\left(x_{n}\right)\right)+H_{d}\left(T\left(u_{n}\right), T\left(x^{*}\right)\right)\right] \leq \\
& \leq s D_{d}\left(u_{n}, T\left(u_{n}\right)\right)+2 s H_{d}^{+}\left(T\left(u_{n}\right), T\left(x^{*}\right)\right) \leq \\
& \leq s D_{d}\left(u_{n}, T\left(x_{n}\right)\right)+2 s k d\left(u_{n}, x^{*}\right) .
\end{aligned}
$$

Hence,

$$
d\left(u_{n}, x^{*}\right) \leq \frac{s}{1-2 s k} D_{d}\left(u_{n}, T\left(u_{n}\right)\right) \rightarrow 0, \text { as } n \rightarrow \infty .
$$

Now we will present the Ulam-Hyers property of the fixed point problem (P).

Definition 10. Let (X,d) be a b-metric space with constant $s \geq 1$ and let $T: X \rightarrow$ $P(X)$ be a multi-valued operator. Let us to consider the inclusion $x \in T(x)$ and the inequality

$$
D_{d}(x, T(x)) \leq \varepsilon \text { where } \varepsilon>0 \text { and } x \in X .
$$

By definition, the inclusion $(\mathrm{P})$ is called generalized Ulam-Hyers stable if and only if, there exists a function $\psi: \mathbb{R}_{+} \rightarrow \mathbb{R}_{+}$increasing, continuous in 0 with $\psi(0)=0$, such that for each $\varepsilon>0$ and for each solution $u^{*} \in X$ of the inequality (3.11), there exists a solution $x^{*} \in X$ of (P) such that,

$$
d\left(u^{*}, x^{*}\right) \leq \psi(\varepsilon) .
$$


Theorem 5. Let $T: X \rightarrow P_{c l}(X)$ be a multi-valued operator which verifies (1) and (3) from Corollary 2 with $k<\frac{1}{2 s}$. Then the fixed point inclusion $(\mathrm{P})$ is Ulam-Hyers stable.

Proof. From Corollary 2 we have that $\mathrm{T}$ has a unique fixed point $x^{*} \in X$ such that $T\left(x^{*}\right)=\left\{x^{*}\right\}$.

Let $\varepsilon>0$ and $u^{*} \in X$ be a $\varepsilon$-solution of the fixed point problem (P), i.e., a solution of the inequality 3.11 .

Then, we have $D_{d}\left(u^{*}, T\left(u^{*}\right)\right) \leq \varepsilon$. Hence, we get

$$
\begin{aligned}
d\left(u^{*}, x^{*}\right) & =D_{d}\left(u^{*}, T\left(x^{*}\right)\right) \leq \\
& \leq s\left[D_{d}\left(u^{*}, T\left(u^{*}\right)\right)+e_{d}\left(T\left(u^{*}\right), T\left(x^{*}\right)\right)\right] \leq \\
& \leq s\left[D_{d}\left(u^{*}, T\left(u^{*}\right)\right)+H_{d}\left(T\left(u^{*}\right), T\left(x^{*}\right)\right)\right] \leq \\
& \leq s D_{d}\left(u^{*}, T\left(u^{*}\right)\right)+2 s H_{d}^{+}\left(T\left(u^{*}\right), T\left(x^{*}\right)\right) \leq \\
& \left.\leq s D_{d}\left(u^{*}, T\left(u^{*}\right)\right)+2 s k d\left(u^{*}, x^{*}\right)\right) .
\end{aligned}
$$

Thus,

$$
d\left(u^{*}, x^{*}\right) \leq \frac{s}{1-2 s k} \varepsilon
$$

Therefore, if we consider $\psi: \mathbb{R}_{+} \rightarrow \mathbb{R}_{+}$, given by $\psi(t)=\operatorname{ct}\left(\right.$ with $\left.c=\frac{s}{1-2 s k}>0\right)$, we can conclude that the inclusion $(\mathrm{P})$ is Ulam-Hyers stable.

Using the global existence result for the fixed point problem( see Corollary 2), we can prove the limit shadowing property of the fixed point problem (P).

Definition 11. Let $(X, d)$ be a metric space with constant $s \geq 1$ and $T: X \rightarrow$ $P_{c l}(X)$ be a multivalued operator. By definition, the fixed problem (P) has the limit shadowing property if, for any sequence $\left(x_{n}\right)_{n \in \mathbb{N}}$ in $\mathrm{X}$ for which,

$$
D_{d}\left(x_{n}, T\left(x_{n}\right)\right) \rightarrow 0 \text { as } n \rightarrow \infty,
$$

there exists a sequence $\left(u_{n}\right)_{n \in \mathbb{N}}$ in $\mathrm{X}$ such that,

$$
d\left(x_{n}, u_{n}\right) \rightarrow 0 \text { as } n \rightarrow \infty .
$$

Theorem 6. Let $(X, d)$ be a metric space with constant $s \geq 1$ and $T: X \rightarrow P_{c l}(X)$ be a multivalued operator which verifies (1) and (3) from Corollary 2. Then, the fixed point problem $(\mathrm{P})$ has the limit shadowing property.

Proof. By Corollary 2 we get that $\operatorname{Fix}(T)=\left\{x^{*}\right\}$ and for any initial point $u_{0} \in$ $X$, there exists a sequence $\left(u_{n}\right)_{n \in \mathbb{N}}$ in X with $u_{n+1} \in T\left(u_{n}\right)$ for all $n \in \mathbb{N}$, such that $\left(u_{n}\right)_{n \in \mathbb{N}} \rightarrow x^{*}$ as $n \rightarrow \infty$. 
Let $\left(x_{n}\right)_{n \in \mathbb{N}}$ be a sequences in $\mathrm{X}$ such that,

$$
D_{d}\left(x_{n+1}, T\left(x_{n}\right)\right) \rightarrow 0 \text { as } n \rightarrow \infty .
$$

Thus, $d\left(x_{n+1}, u_{n+1}\right) \leq s\left[d\left(x_{n+1}, x^{*}\right)+d\left(x^{*}, u_{n+1}\right)\right]$.

Next, we can write,

$$
\begin{aligned}
d\left(x_{n+1}, x^{*}\right) & =D_{d}\left(x_{n+1}, T\left(x^{*}\right)\right) \leq \\
& \leq s\left[D_{d}\left(x_{n+1}, T\left(x_{n}\right)\right)+e_{d}\left(T\left(x_{n}\right), T\left(x^{*}\right)\right] \leq\right. \\
& \leq s\left[D_{d}\left(x_{n+1}, T\left(x_{n}\right)\right)+H_{d}\left(T\left(x_{n}\right), T\left(x^{*}\right)\right] \leq\right. \\
& \leq s\left[D_{d}\left(x_{n+1}, T\left(x_{n}\right)\right)+2 H_{d}^{+}\left(T\left(x_{n}\right), T\left(x^{*}\right)\right] \leq\right. \\
& \leq s D_{d}\left(x_{n+1}, T\left(x_{n}\right)\right)+2 k s d\left(x_{n}, x^{*}\right) .
\end{aligned}
$$

We denote $2 k s=\alpha<1$, thus

$$
\begin{aligned}
d\left(x_{n+1}, x^{*}\right) & \leq s D_{d}\left(x_{n+1}, T\left(x_{n}\right)\right)+\alpha d\left(x_{n}, x^{*}\right) \leq \\
& \leq s D_{d}\left(x_{n+1}, T\left(x_{n}\right)\right)+\alpha\left[s D_{d}\left(x_{n}, T\left(x_{n-1}\right)\right)+\alpha d\left(x_{n-1}, x^{*}\right)\right] \\
& =s\left[D_{d}\left(x_{n+1}, T\left(x_{n}\right)\right)+D_{d}\left(x_{n}, T\left(x_{n-1}\right)\right)\right]+\alpha^{2} d\left(x_{n-1}, x^{*}\right) \\
& \leq \cdots \leq \\
& \leq s\left[D_{d}\left(x_{n+1}, T\left(x_{n}\right)\right)+\ldots+\alpha^{n} D_{d}\left(x_{1}, T\left(x_{0}\right)\right)\right]+\alpha^{n} d\left(x_{0}, x^{*}\right) .
\end{aligned}
$$

By Cauchy's Lemma 4 we obtain that,

$$
D_{d}\left(x_{n+1}, T\left(x_{n}\right)\right)+\ldots+\alpha^{n} D_{d}\left(x_{1}, T\left(x_{0}\right)\right) \rightarrow 0 \text { as } n \rightarrow \infty .
$$

Thus, we obtain that $d\left(x_{n}, x^{*}\right) \rightarrow 0$ as $n \rightarrow \infty$.

Then, $d\left(x_{n+1}, u_{n+1}\right) \rightarrow 0$ as $n \rightarrow \infty$.

\section{EXTENDED COUPLED FIXED POINT THEOREMS FOR MULTI-VALUED CONTRACTIONS WITH RESPECT TO A POMPEIU TYPE METRIC}

The purpose of this section is to give some extended coupled fixed point theorems for multi-value operators satisfying a contraction type condition with respect to the excess functional. We will consider here the context of a b-metric space. Our results are new even for the case of metric spaces and they extend some theorems given in [8], [16], [5] and [19] and other papers in the literature.

Theorem 7. Let $(X, \preceq, d),(Y, \preceq, d)$ be two ordered complete $b$-metric spaces with constant $s \geq 1$. Let $T_{1}: X \times Y \rightarrow P_{c l}(X)$ and $T_{2}: X \times Y \rightarrow P_{c l}(Y)$ be two multi-valued operators with closed graph and having the strict mixed monotone property with respect to " $\preceq "$. Assume that:

(1) there exist $k_{1}, k_{2}>0$ with $k_{1}+k_{2} \in(0,1)$ such that:

$$
e_{d}\left(T_{1}(x, y), T_{1}(u, v)\right)+e_{d}\left(T_{1}(u, v), T_{1}(x, y)\right) \leq k_{1}[d(x, u)+\rho(y, v)],
$$




$$
\begin{aligned}
& \forall x \preceq u \text { and } y \succeq v, \\
& e_{\rho}\left(T_{2}(x, y), T_{2}(u, v)\right)+e_{\rho}\left(T_{2}(u, v), T_{2}(x, y)\right) \leq k_{2}[d(x, u)+\rho(y, v)], \\
& \forall x \preceq u \text { and } y \succeq v,
\end{aligned}
$$

(2) there exists $\left(x_{0}, y_{0}\right) \in X \times Y$ such that

$$
x_{0} \preceq_{w k} T_{1}\left(x_{0}, y_{0}\right), y_{0} \succeq_{w k} T_{2}\left(x_{0}, y_{0}\right)
$$

or

$$
x_{0} \succeq_{w k} T_{1}\left(x_{0}, y_{0}\right), y_{0} \preceq_{w k} T_{2}\left(x_{0}, y_{0}\right) .
$$

Then, the following conclusions hold:

(i) there exist $\left(x^{*}, y^{*}\right) \in X \times Y$ and the sequences $\left(x_{n}\right)_{n \in \mathbf{N}}$ in $X$ and $\left(y_{n}\right)_{n \in \mathbf{N}}$ in $Y$ defined by

$$
\left\{\begin{array}{l}
x_{n+1} \in T_{1}\left(x_{n}, y_{n}\right) \\
y_{n+1} \in T_{2}\left(x_{n}, y_{n}\right)
\end{array} \quad \text { for all } n \in \mathbf{N},\right.
$$

such that $x_{n} \rightarrow x^{*}$ and $y_{n} \rightarrow y^{*}$ as $n \rightarrow \infty$ and

$$
\left\{\begin{array}{l}
x^{*} \in T_{1}\left(x^{*}, y^{*}\right) \\
y^{*} \in T_{2}\left(x^{*}, y^{*}\right)
\end{array} .\right.
$$

(ii) If we denote $\tilde{d}\left(z_{n}, z^{*}\right)=d\left(x_{n}, x^{*}\right)+\rho\left(y_{n}, y^{*}\right)$ and $\tilde{d}\left(z_{0}, z_{1}\right)=d\left(x_{0}, x_{1}\right)+\rho\left(y_{0}, y_{1}\right)$ then we have the following estimation holds,

$$
\tilde{d}\left(z_{n+1}, z^{*}\right) \leq \frac{\beta^{n} S s}{1-\beta} \tilde{d}\left(z_{0}, z_{1}\right) \text {, where } \beta>0 \text { is any number with } \beta<\frac{k_{1}+k_{2}}{2} \text {. }
$$

Proof. Let $Z=X \times Y, T: Z \rightarrow P_{c l}(Z), T(x, y)=T_{1}(x, y) \times T_{2}(x, y)$ and consider the functional $\tilde{d}: Z \times Z \rightarrow \mathbf{R}_{+}, \tilde{d}((x, y),(u, v))=d(x, u)+\rho(y, v)$.

Then, for $z_{0}=\left(x_{0}, y_{0}\right) \in Z$, there exist $x_{1} \in T_{1}\left(x_{0}, y_{0}\right)$ and $y_{1} \in T_{2}\left(x_{0}, y_{0}\right)$ such that

$$
x_{0} \preceq x_{1} \text { and } y_{0} \succeq y_{1} \Rightarrow\left(x_{0}, y_{0}\right) \preceq_{p}\left(x_{1}, y_{1}\right) \Rightarrow z_{0} \preceq_{p} z_{1} \text {. }
$$

Thus, $z_{0} \preceq_{p} T\left(z_{0}\right)$.

On the other hand, for $z=(x, y) \in Z$ and $w=(u, v) \in T(Z)$ with $z \preceq_{p} w$ we get

$$
\begin{aligned}
& D_{\tilde{d}}(w, T(w))=D_{\tilde{d}}\left((u, v), T_{1}(u, v) \times T_{2}(u, v)\right)= \\
& \quad=D_{d}\left(u, T_{1}(u, v)\right)+D_{\rho}\left(v, T_{2}(u, v)\right) \leq \\
& \quad \leq e_{d}\left(T_{1}(x, y), T_{1}(u, v)\right)+e_{\rho}\left(T_{2}(x, y), T_{2}(u, v)\right)= \\
& \quad=2 H_{d}^{+}\left(T_{1}(x, y) \times T_{2}(x, y), T_{1}(u, v) \times T_{2}(u, v)\right)=2 H_{\tilde{d}}^{+}(T(z), T(w)) .
\end{aligned}
$$

Hence, we conclude that

$$
D_{\tilde{d}}(w, T(w)) \leq 2 H_{\tilde{d}}^{+}(T(z), T(w)),
$$

for all $z \in Z$ and $w \in T(z)$ with $z \leq_{p} w$. 
Now, we proof that:

$$
\begin{aligned}
& H_{\tilde{d}}^{+}(T(z), T(w)) \leq k \tilde{d}(z, w) \forall z, w \in Z z \leq{ }_{p} w . \\
& H_{\tilde{d}}^{+}(T(z), T(w))= H_{\tilde{d}}^{+}\left(T_{1}(z) \times T_{2}(z), T_{1}(w) \times T_{2}(w)\right)= \\
&= H_{d}^{+}\left(T_{1}(z), T_{1}(w)\right)+H_{d}^{+}\left(T_{2}(z), T_{2}(w)\right)= \\
&= \frac{1}{2}\left(e_{d}\left(T_{1}(x, y), T_{1}(u, v)\right)+e_{d}\left(T_{1}(u, v), T_{1}(x, y)\right)\right)+ \\
&+\frac{1}{2}\left(e_{\rho}\left(T_{2}(x, y), T_{2}(u, v)\right)+e_{\rho}\left(T_{2}(u, v), T_{2}(x, y)\right)\right) \leq \\
& \leq \frac{1}{2} k_{1}[d(x, u)+\rho(y, v)]+\frac{1}{2} k_{2}[d(x, u)+\rho(y, v)]= \\
&= \frac{1}{2}\left(k_{1}+k_{2}\right)[d(x, u)+\rho(y, v)]=\frac{1}{2}\left(k_{1}+k_{2}\right) \tilde{d}(z, w) .
\end{aligned}
$$

Hence, there exists $k:=\frac{1}{2}\left(k_{1}+k_{2}\right) \in(0,1)$ such that

$$
H_{\tilde{d}}^{+}(T(z), T(w)) \leq k \tilde{d}(z, w) \forall z, w \in Z z \leq_{p} w .
$$

We will show now that $\mathrm{T}$ is an increasing operator, i.e, if $z \leq_{p} w$ we have $T(z) \leq_{s}$ $t T(w)$. This is equivalent to the following relations

$$
T_{1}(x, y) \times T_{2}(x, y) \leq_{s t} T_{1}(u, v) \times T_{2}(u, v)
$$

or

$$
T_{1}(x, y) \leq_{s t} T_{1}(u, v) \text { and } T_{2}(x, y) \leq_{s t} T_{2}(u, v) .
$$

We have

$$
\begin{aligned}
& x \preceq u \Rightarrow\left\{\begin{array}{l}
T_{1}(x, y) \leq_{s t} T_{1}(u, y), \forall y \in Y \\
T_{2}(x, y) \geq_{s t} T_{2}(u, y), \forall y \in Y
\end{array},\right. \\
& y \succeq v \Rightarrow\left\{\begin{array}{l}
T_{1}(x, y) \leq_{s t} T_{1}(x, v), \forall x \in X \\
T_{2}(x, y) \geq_{s t} T_{2}(x, v), \forall x \in X
\end{array},\right.
\end{aligned}
$$

If in the relation (4.4) we put $x:=u$, then we have $T_{1}(u, y) \leq_{s t} T_{1}(u, v)$. Using the relation (4.3) we get $T_{1}(x, y) \leq_{s t} T_{1}(u, v)$.

On the other hand, for $x:=u$ in the relation (4.4) we get $T_{2}(u, y) \geq_{s t} T_{2}(u, v)$. Using the relation (4.3) we obtain $T_{2}(x, y) \geq_{s t} T_{2}(u, v)$. Thus $T: Z \rightarrow Z, T(x, y)=$ $T_{1}(x, y) \times T_{2}(x, y)$ is an increasing operator.

Thus, the operator $T: Z \rightarrow Z$ has the following properties:

(1) $\mathrm{T}$ is increasing on $\mathrm{Z}$.

(2) There exists $z_{0}=\left(x_{0}, y_{0}\right) \in Z$ such that $z_{0} \leq_{p} T\left(z_{0}\right)$. 
(3) There exists $k \in\left(0, \frac{1}{s}\right)$ such that

$$
H_{\tilde{d}}^{+}(T(z), T(w)) \leq k \tilde{d}(z, w) \forall z, w \in Z z \leq_{p} w .
$$

(4) For every $z=(x, y) \in Z$ and $w=(u, v) \in T(z)$ with $z \leq_{p} w$, we have

$$
D_{\tilde{d}}(w, T(w)) \leq 2 H_{\tilde{d}}^{+}(T(z), T(w)) .
$$

Thus, by Theorem 1 we get that $\operatorname{Fix}(T) \neq \varnothing$ and there exists a sequence $\left(z_{n}\right)_{n \in \mathbb{N}} \subset$ $Z, z_{n}=\left(x_{n}, y_{n}\right) \in X \times Y$ of successive approximation of T starting from $z_{0} \in Z$ which converges to a fixed point $z^{*}=\left(x^{*}, y^{*}\right)$ of $\mathrm{T}$.

Thus,

$$
z^{*} \in T\left(z^{*}\right) \Rightarrow\left(x^{*}, y^{*}\right) \in T\left(x^{*}, y^{*}\right) \Rightarrow\left(x^{*}, y^{*}\right) \in T_{1}\left(x^{*}, y^{*}\right) \times T_{2}\left(x^{*}, y^{*}\right)
$$

which leads to

$$
\left\{\begin{array}{l}
x^{*} \in T_{1}\left(x^{*}, y^{*}\right) \\
y^{*} \in T_{2}\left(x^{*}, y^{*}\right) .
\end{array}\right.
$$

By Theorem 1 (ii) we get that

$$
\tilde{d}\left(z_{n+1}, z^{*}\right) \leq \frac{\beta^{n} S s}{1-\beta} \tilde{d}\left(z_{0}, z_{1}\right) \text {, where } \beta<\frac{k_{1}+k_{2}}{2} .
$$

Corollary 3. Let $(X, \preceq, d),(Y, \preceq, d)$ be two orderd complete $b$-metric spaces with constant $s \geq 1$. Let $T_{1}: X \times Y \rightarrow P_{c l}(X)$ and $T_{2}: X \times Y \rightarrow P_{c l}(Y)$ be two multi-valued operators having the strict mixed monotone property with respect to " $\preceq "$. Assume that, there exist $k_{1}, k_{2}>0$ with $k_{1}+k_{2} \in(0,1)$ such that:

$$
\begin{aligned}
& e_{d}\left(T_{1}(x, y), T_{1}(u, v)\right)+e_{d}\left(T_{1}(u, v), T_{1}(x, y)\right) \leq k_{1}[d(x, u)+\rho(y, v)], \\
& \forall(x, y) \in(X \times Y), \\
& e_{\rho}\left(T_{2}(x, y), T_{2}(u, v)\right)+e_{\rho}\left(T_{2}(u, v), T_{2}(x, y)\right) \leq k_{2}[d(x, u)+\rho(y, v)], \\
& \forall(x, y) \in(X \times Y) .
\end{aligned}
$$

Then, the following conclusions hold:

a) there exists $\left(x^{*}, y^{*}\right) \in X \times Y$ such that the sequence $\left(x_{n}\right)_{n \in \mathbf{N}}$ in $X$ and $\left(y_{n}\right)_{n \in \mathbf{N}}$ in $Y$ with,

$$
\left\{\begin{array}{l}
x_{n+1} \in T_{1}\left(x_{n}, y_{n}\right) \\
y_{n+1} \in T_{2}\left(x_{n}, y_{n}\right)
\end{array} \quad \text { for all } n \in \mathbf{N}\right.
$$

such that $x_{n} \rightarrow x^{*}$ and $y_{n} \rightarrow y^{*}$ as $n \rightarrow \infty$ and

$$
\left\{\begin{array}{l}
x^{*} \in T_{1}\left(x^{*}, y^{*}\right) \\
y^{*} \in T_{2}\left(x^{*}, y^{*}\right)
\end{array} .\right.
$$


b) If we denote $\tilde{d}\left(z_{n}, z^{*}\right)=d\left(x_{n}, x^{*}\right)+\rho\left(y_{n}, y^{*}\right)$ and $\tilde{d}\left(z_{0}, z_{1}\right)=d\left(x_{0}, x_{1}\right)+\rho\left(y_{0}, y_{1}\right)$, then we have the following estimation holds,

$$
\tilde{d}\left(z_{n+1}, z^{*}\right) \leq \frac{\beta^{n} S s}{1-\beta} \tilde{d}\left(z_{0}, z_{1}\right) \text {, where } \beta>0 \text { is any number with } \beta<\frac{k_{1}+k_{2}}{2} \text {. }
$$

The following result is about the uniqueness of the extended coupled fixed point.

Theorem 8. In addition to the hypotheses of the Corollary 3 we suppose that:

(1) there exists $\left(x^{*}, y^{*}\right) \in X \times Y$ such that

$$
\left\{\begin{array}{l}
T_{1}\left(x^{*}, y^{*}\right)=\left\{x^{*}\right\} \\
T_{2}\left(x^{*}, y^{*}\right)=\left\{y^{*}\right\}
\end{array} .\right.
$$

(2) for every $(\bar{x}, \bar{y}) \in \operatorname{CFix}\left(T_{1}, T_{2}\right)$ we have $\bar{x} \leq x^{*}$ and $\bar{y} \geq y^{*}$ or $x^{*} \leq \bar{x}$ and $y^{*} \geq \bar{y}$.

Then, we obtain that the extended coupled fixed point problem (P1) has an unique solution.

Proof. Let $Z:=X \times Y, T: Z \rightarrow P_{c l}(Z), T(x, y)=T_{1}(x, y) \times T_{2}(x, y)$ and consider the functional $\tilde{d}: Z \times Z \rightarrow \mathbb{R}_{+}, \tilde{d}((x, y),(u, v))=d(x, u)+\rho(y, v)$.

Let $z^{*} \in Z, z^{*}=\left(x^{*}, y^{*}\right)$ such that $T\left(z^{*}\right)=\left\{z^{*}\right\}$ and $\bar{z}=(\bar{x}, \bar{y}) \in Z$ such that $\bar{z} \in$ Fix $(T)$ with $\bar{z} \leq z^{*}$ or $z^{*} \leq \bar{z}$.

By Theorem 2 we get that Fix $(T)=\operatorname{SFix}(T)=\left\{z^{*}\right\}$. Hence CFix $\left(T_{1}, T_{2}\right)=\left\{z^{*}\right\}$.

In the next part of this section, we will present some properties of the extended coupled fixed point problem (P1).

The first result is a data dependence problem for the extended coupled fixed point problem (P1).

Theorem 9. Let $(X, d),(Y, d)$ be two coupled b-metric spaces with constant $s \geq 1$. Let $T_{1}: X \times Y \rightarrow P_{c l}(X), T_{2}: X \times Y \rightarrow P_{c l}(Y), S_{1}: X \times Y \rightarrow P(X)$ and $S_{2}: X \times Y \rightarrow$ $P(Y)$ be four multi-valued operators. We suppose that:

(1) there exist $k_{1}, k_{2}>0$ with $k_{1}+k_{2} \in(0,1)$ such that:

$$
\begin{aligned}
& e_{d}\left(T_{1}(x, y), T_{1}(u, v)\right)+e_{d}\left(T_{1}(u, v), T_{1}(x, y)\right) \leq k_{1}[d(x, u)+\rho(y, v)], \forall(x, y) \in X \times Y, \\
& e_{\rho}\left(T_{2}(x, y), T_{2}(u, v)\right)+e_{\rho}\left(T_{2}(u, v), T_{2}(x, y)\right) \leq k_{2}[d(x, u)+\rho(y, v)], \forall(x, y) \in X \times Y,
\end{aligned}
$$

(2) there exists $\left(x^{*}, y^{*}\right) \in X \times Y$ such that

$$
\left\{\begin{array}{l}
T_{1}\left(x^{*}, y^{*}\right)=\left\{x^{*}\right\} \\
T_{2}\left(x^{*}, y^{*}\right)=\left\{y^{*}\right\}
\end{array} .\right.
$$


(3) there exists $\left(u^{*}, v^{*}\right) \in X \times Y$ such that

$$
\left\{\begin{array}{l}
u^{*} \in S_{1}\left(u^{*}, v^{*}\right) \\
v^{*} \in S_{2}\left(u^{*}, v^{*}\right)
\end{array} .\right.
$$

(4) there exists $\eta>0$ such that

$$
e_{\tilde{d}}\left(T_{1}(x, y) \times T_{2}(x, y), S_{1}(x, y) \times S_{2}(x, y)\right) \leq \eta, \forall(x, y) \in X \times Y .
$$

Then, $e_{\tilde{d}}\left(\operatorname{CFix}\left(S_{1}, S_{2}\right), \operatorname{CFix}\left(T_{1}, T_{2}\right)\right) \leq \frac{s \eta}{1-2 s k}$.

Proof. Let $Z:=X \times Y, T: Z \rightarrow P_{c l}(Z), T(x, y)=T_{1}(x, y) \times T_{2}(x, y)$ and consider the functional $\tilde{d}: Z \times Z \rightarrow \mathbb{R}_{+}, \tilde{d}((x, y),(u, v))=d(x, u)+\rho(y, v)$.

Let $z^{*} \in Z, z^{*}=\left(x^{*}, y^{*}\right)$ such that $T\left(z^{*}\right)=\left\{z^{*}\right\}$.

We consider $S: Z \rightarrow P(Z), S(x, y)=S_{1}(x, y) \times S_{2}(x, y)$. Let $w^{*} \in Z, w^{*}=\left(u^{*}, v^{*}\right)$ such that $w^{*} \in S\left(w^{*}\right)$.

By Theorem 3 for the multi-valued operators $\mathrm{T}$ and $\mathrm{S}$ we obtain that

$$
d\left(w^{*}, z^{*}\right) \leq \frac{s \eta}{1-2 s k} \text { where } k:=\frac{k_{1}+k_{2}}{2} .
$$

Since $w^{*} \in C F i x(S)$ are arbitrary chosen, we obtain that

$$
e_{\tilde{d}}\left(\operatorname{CFix}\left(S_{1}, S_{2}\right), \operatorname{CFix}\left(T_{1}, T_{2}\right)\right) \leq \frac{s \eta}{1-2 s k} .
$$

The second problem we intend to study is the well-posedness of the extended coupled fixed point problem.

Definition 12. We consider the extended coupled fixed point problem (P1). By definition, (P1) is well-posed for $T_{1}$ and $T_{2}$ with respect to $D_{d}$ if:

(1) $\operatorname{CFix}\left(T_{1}, T_{2}\right)=\left\{\left(u^{*}, v^{*}\right)\right\},\left(u^{*}, v^{*}\right) \in X \times Y$,

(2) if there exists a sequences $\left(u_{n}, v_{n}\right) \in X \times Y$ with

$$
\left\{\begin{array}{l}
D_{d}\left(u_{n}, T_{1}\left(u_{n}, v_{n}\right)\right) \rightarrow 0 \\
D_{d}\left(v_{n}, T_{2}\left(u_{n}, v_{n}\right)\right) \rightarrow 0
\end{array}\right.
$$

then $d\left(u_{n}, u^{*}\right)+d\left(v_{n}, v^{*}\right) \rightarrow 0$ as $n \rightarrow \infty$.

Theorem 10. We suppose that all the hypotheses of Theorem 9 take place. Then the extended coupled fixed problem (P1) is well-posed for $T_{1}$ and $T_{2}$ with respect to $D_{d}$. 
Proof. Let $Z:=X \times Y, T: Z \rightarrow P_{c l}(Z), T(x, y)=T_{1}(x, y) \times T_{2}(x, y)$ and consider the functional $\tilde{d}: Z \times Z \rightarrow \mathbb{R}_{+}, \tilde{d}((x, y),(u, v))=d(x, u)+\rho(y, v)$.

Since the multi-valued operator $T$ satisfies the hypotheses of Corollary 9 , we get that $\mathrm{T}$ has a unique extended coupled fixed point. Let $z^{*} \in Z, z^{*}=\left(x^{*}, y^{*}\right)$ such that $T\left(z^{*}\right)=\left\{z^{*}\right\}$

Let $w_{n}=\left(u_{n}, v_{n}\right) \in Z$, with

$$
\left\{\begin{array}{l}
D_{d}\left(u_{n}, T_{1}\left(u_{n}, v_{n}\right)\right) \rightarrow 0 \\
D_{d}\left(v_{n}, T_{2}\left(u_{n}, v_{n}\right)\right) \rightarrow 0
\end{array} .\right.
$$

By Theorem 4 for the multi-valued operator $\mathrm{T}$ we obtained that $d\left(w_{n}, z^{*}\right) \rightarrow 0$, as $n \rightarrow \infty$.

\section{REFERENCES}

[1] I. Bakhtin, "The contraction mapping principle in almost metric spaces," Funct. Anal., vol. 30, pp. 26-37, 1989.

[2] V. Berinde, "Generalized coupled fixed point theorems for mixed monotone mappings in partially ordered metric spaces," Nonlinear Anal., vol. 74, no. 18, pp. 7347-7355, 2011.

[3] M. Boriceanu-Bota, A. Petruşel, G. Petruşel, and B. Samet, "Coupled fixed point theorems for single-valued operators in b-metric spaces," Fixed Point Theory Appl., 2015, doi: 10.1186/s13663-015-0482-3.

[4] M. Boriceanu-Bota, A. Petruşel, and I. A. Rus, "Fixed point theorems for some multi-valued generalized contractions in b-metric spaces," International J. Math. Statistics, vol. 6, pp. 65-76, 2010 .

[5] I. Coroian, "Fixed points for multivalued contractions with respect to a Pompeiu type," J. Nonlinear Sci. Appl, vol. 10, pp. 6084-6101, 2017.

[6] H. Covitz and S. B. Nadler, "Multi-valued contraction mappings in generalized metric spaces," Israel J. Math., vol. 8, pp. 5-11, 1970.

[7] H. Covitz and S. B. Nadler, "Nonlinear set-valued contraction mappings in B-metric spaces," Atti Sem. Mat. Univ. Modena, vol. 46, pp. 263-276, 1998.

[8] T. Gnana Bhaskar and V. Lakshmikantham, "Fixed point theorems in partially ordered metric spaces and applications," Nonlinear Anal., vol. 65, pp. 1379-1393, 2006.

[9] C. Himmelberg and F. Van Vleck, "Lipschitzian generalized differential equations," Rend. Sem. Mat. Univ. Padova, vol. 48, pp. 159-169, 1972.

[10] W. Kirk and N. Shahzad, "Remarks on metric transforms and fixed-point theorems." Fixed Point Theory and Appl., vol. 106, pp. 1-11, 2013.

[11] W. Kirk and N. Shahzad, Fixed Point Theory in Distance Spaces. New York: Springer Heidelberg, 2014.

[12] V. Lakshmikantham and L. Ćirić, "Coupled fixed point theorems for nonlinear contractions in partially ordered metric spaces." Nonlinear Anal., vol. 70, pp. 4341-4349, 2009.

[13] R. Miculescu and A. Mihail, "New fixed point theorems for set-valued contractions in b-metric spaces." J. Fixed Point Theory Appl., vol. 19, pp. 4341-4349, 2017.

[14] S. Nadler, "Multi-valued contraction mappings." Pacific J. Math., vol. 30, pp. 475-488, 1969.

[15] H. Pathak and N. Shahzad, "A generalization of Nadlers fixed point theorem and its application to nonconvex integral inclusions." Topol. Methods Nonlinear Anal., vol. 41, pp. 207-227, 2013. 
[16] A. Petruşel, "Multi-valued weakly Picard operators and applications." Sci. Math. Japon., vol. 59, pp. 169-202, 2004.

[17] A. Petruşel, G. Petruşel, B. Samet, and J.-C. Yao, "Coupled fixed point theorems for symmetric multi-valued contractions in b-metric space with applications to systems of integral inclusions." J. Nonlinear Convex Anal., vol. 17, no. 7, pp. 1265-1282, 2016.

[18] A. Petruşel, G. Petruşel, and J.-C. Yao, "A study of a system of operator inclusions via a fixed point approach and applications to functional-differential inclusions." Carpathian J. Math., vol. 32, no. 3, pp. 349-361, 2016.

[19] T. Wang, "Fixed point theorems and fixed point stability for multivalued mappings on metric spaces,” Nanjing Daxue Xuebao Shuxue Bannian Kan, vol. 6, no. 1, pp. 16-23, 1989.

Author's address

Iulia Coroian

University of Agricultural Sciences and Veterinary Medicine, Calea Mănăştur Street, 3-5, 400372, Cluj-Napoca, Romania.

E-mail address: coroian.iuliaegmail.com 